\title{
CAMBIO FAMILIAR Y MALTRATO CONYUGAL A LA MUJER
}

\author{
GERARDO MEIL LANDWERLIN \\ Universidad Autónoma de Madrid
}

\author{
PALABRAS CLAVE ADICIONALES \\ ADDITIONAL KEYWORDS \\ Violencia doméstica, Matrimonio, Uniones de \\ Partner Violence, Marriage, Cohabitation. \\ hecho.
}

RESUMEN. Según la teoría de los recursos, la ganancia de recursos de poder por parte de las mujeres derivada de las distintas dimensiones del cambio familiar (desintitucionalización de la familia, incorporación de la mujer al mercado de trabajo, mayor nivel educativo, etc.) ha comportado un mayor poder de negociación del contenido de los roles familiares y domésticos. Esta ganancia en recursos de poder debería haberse traducido en una menor violencia conyugal contra las mujeres que viven en uniones "modernas". A partir de la macroencuesta sobre violencia de género realizada por el Instituto de la Mujer en 1999 se analiza si las distintas dimensiones del cambio familiar están asociadas con un menor riesgo de maltrato. Los resultados obtenidos mediante ajustes de regresión logística indican que el cambio familiar y el correspondiente aumento de poder de negociación de las mujeres no se ha traducido automáticamente en una reducción del riesgo de maltrato.

\begin{abstract}
According to family resources theory, the empowerment of women caused by the different dimensions of family change during the last decades (deinstitutionalization of the family, female work, higher education, etc) has brought about a greater say in the process of negotiation of gender roles inside the family and the couple life. This impowerment of women should have implied also a lower degree of partner violence against women in "modern" family life settings. Based on a survey on violence against women carried out in 1999 in Spain among 20.000 women, the paper analyses using logistic regressions models if different dimensions of family change (female work, cohabitation, among others) are associated with lower risks of partner violence against women. Results from such type of analyses show that there is little support to suppose that family change and the corresponding empowerment of women which it implies will bring about a lower risk of partner violence against women.
\end{abstract}

E-mail: gerardo.meil@uam.es

\section{Revista Internacional de Sociología (RIS)}

Tercera Época, nº 37, Enero-Abril, 2004, pp. 7-27. 
RIS

REVISTA INTERNACIONAL DE SOCIOLOCIA

№ 37, ENERO-ABRIL, 2004

GERARDO MEIL LANDWERLIN

\section{INTRODUCCIÓN}

Actualmente se está asistiendo a una creciente toma de conciencia de que la vida familiar no sólo representa un modelo de felicidad y bienestar para sus miembros, sino que también está permeada por el uso de la violencia de unos miembros contra otros. De hecho, entre dos de cada tres y tres de cada cuatro encuestados recientemente por el Centro de Investigaciones Sociológicas creen que las agresiones o malos tratos físicos hacia las mujeres y hacia los hijos, así como los abusos sexuales, son bastante o muy frecuentes. El uso de la violencia en el espacio doméstico no es un fenómeno nuevo derivado de un individuąlismo creciente en las sociedades altamente desarrolladas y de una supuesta descomposición de la familia, sino que multitud de indicadores evidencian que ha existido a lo largo de todos los tiempos. Así, por ejemplo, el infanticidio, particularmente el practicado con niñas, niños con discapacidades o con los hijos no matrimoniales, ha sido un fenómeno ampliamente extendido en el pasado. Por otro lado, la aplicación de la violencia física sobre los niños en el proceso educativo según la máxima de "la letra con sangre entra" ha sido hasta no hace mucho tiempo el modelo educativo por excelencia, y el pegar a las esposas, una forma legítima de "poner orden en casa". De hecho, el patriarcado como forma de organización de las relaciones familiares supone la licencia para pegar como fórmula para mantener "el orden" o simplemente para descargar tensiones y frustraciones acumuladas fuera del espacio familiar. Lo novedoso de la violencia doméstica no deriva, así, de su existencia, sino de la toma de conciencia de su existencia, así como, sobre todo, de la creciente pérdida de "comprensión" de la que ha gozado hasta recientemente en casi todas las instancias de las sociedades desarrolladas y del rechazo social activo que de forma creciente concita. En este sentido, no se trata tanto de que no se supiera que dentro del espacio doméstico se utilizaba la fuerza física para controlar los comportamientos, como del hecho de que estos comportamientos hayan dejado de considerarse un asunto privado para convertirse en algo socialmente condenable y también legalmente punible, hasta convertirse en un problema social. En el imaginario colectivo, la familia está dejando de ser considerada sólo en sus aspectos positivos como espacio de amor y solidaridad desinteresada frente a un mundo despersonalizado, competitivo e interesado, para ser vista de una forma más realista, considerando también sus aspectos negativos y sus riesgos potenciales.

Varios son los factores que han contribuido en las últimas décadas a despojar a la violencia doméstica de su carácter privado para convertirla en un problema social. Por lo que se refiere a la violencia contra las mujeres, el principal motor que ha elevado la violencia doméstica a la categoría de problema social ha sido el movimiento feminista, que en el contexto general de la lucha por la igualdad y la no discriminación en función del género ha ido denunciando y desenmascarando las relaciones de poder entre los sexos y sus consecuencias en todos los ámbitos de 
la vida social e individual para las mujeres. El desarrollo y el poder transformador de este movimiento, sin embargo, no habría sido posible si no se hubiesen dado determinadas condiciones socioculturales que han hecho que las denuncias no sólo hayan podido expresarse, sino que hayan sido "escuchadas" transformando la realidad social al pasar a formar parte de los valores sociales básicos de las sociedades contemporáneas, de los programas de los partidos políticos y de las políticas públicas, no sólo ya nacionales, sino incluso de los organismos internacionales. Uno de estos factores es la transformación del modelo de relaciones familiares desde un modelo de familia tradicional con una fuerte jerarquización de los roles familiares y domésticos en función de la edad y el sexo (familia patriarcal), a un modelo más "democrático" en el sentido de una equiparación legal de los derechos y obligaciones de los cónyuges, y una práctica más consensuada en la toma de decisiones en todos los ámbitos del espacio doméstico.

En este contexto sería de esperar que en aquellos modelos de organización familiar con una definición de los roles más negociada, la legitimidad del maltrato hubiera desaparecido y, por tanto, que su extensión fuera menor que en el modelo familiar patriarcal tradicional. Desde esta perspectiva sería de esperar que el cambio familiar no sólo facilitara el cuestionamiento social del maltrato doméstico e, indirectamente, el desarrollo de medidas de intervención social en este sentido, sino que por sí mismo, por el cambio de valores que comporta y por el mayor poder de negociación del orden y de las relaciones domésticas que conlleva para las mujeres (su empowerment en terminología inglesa), contribuyera decisivamente a reducir el riesgo de maltrato hacia las mujeres y, en definitiva, a hacerlo menos frecuente entre las parejas con unos modelos y unas prácticas del rol de género no tradicionales. En lo que sigue queremos abordar la cuestión de si efectivamente cabe esperar una erosión del maltrato doméstico y, más en concreto, del maltrato conyugal, como consecuencia del proceso de cambio familiar que está conociendo la sociedad española.

\section{METODOLOGÍA Y OPERACIONALIZACIÓN DE LAS HIPÓTESIS}

La medición del alcance del maltrato doméstico es, como han señalado múltiples autores (Gelles y Straus, 1979; Villavicencio y Sebastián, 1999; Alberdi y Matas, 2002), muy problemática, tanto por motivos metodológicos y conceptuales, como por la propia predisposición de la población a reconocerse tanto a sí misma como hacia los demás el ser objeto o sujeto de maltrato, a lo que hay que añadir, además, el escaso rigor con el que son elaboradas muchas de las estadísticas disponibles. Básicamente existen dos metodologías diferentes para medir la incidencia del maltrato. Por un lado, se encuentran los registros estadísticos elaborados por las instituciones públicas encargadas legalmente de ello y que se basan bien en las muertes violentas identificadas como causadas por malos tratos, bien en las 
denuncias presentadas ante las fuerzas de seguridad por malos tratos. Junto a estos datos habría que considerar igualmente las estadísticas de las casas de acogida, que no siempre recogen a mujeres que han realizado la correspondiente denuncia. Estos datos, sin embargo, sólo proporcionan una visión muy limitada del alcance del fenómeno, pues sólo en un número limitado de casos se llegan a presentar denuncias. Una forma alternativa de medir la incidencia del maltrato doméstico son las encuestas dirigidas a la población en su conjunto o a un determinado subgrupo de la misma en las que se pregunta a los encuestados si consideran que han sido objeto de malos tratos, o si han sido objeto de determinado tipo de comportamientos definidos por el investigador como tal, o si conocen casos de maltrato. Esta técnica de la encuesta lleva aplicándose en Estados Unidos desde hace más de 20 años para analizar el alcance y las características de la violencia entre los cónyuges (Gelles y Straus, 1979), pero en España sólo se ha comenzado a aplicar recientemente para medir el alcance de la violencia contra las mujeres, pero no así contra los niños, (para los que se dispone sólo de datos sobre denuncias o casos tratados por los servicios sociales) ni contra los hombres o los mayores.

El presente trabajo se basa en la encuesta encargada por el Instituto de la Mujer en 1999 en el marco del segundo Plan de Lucha contra la Violencia Doméstica y realizada por la empresa Sigma 2 a una muestra de 20.552 mujeres mayores de 18 años. La encuesta se realizó telefónicamente, lo que lleva a suponer una menor inhibición a la hora de reconocer situaciones de maltrato y, en consecuencia, una medición más ajustada a la realidad del alcance del fenómeno. Conviene recordar en este contexto que las estimaciones sobre el alcance del fenómeno evidencian grados de incidencia muy diferentes según sean las formulaciones de las preguntas, los tamaños muestrales y la forma de aplicación de la encuesta. Así, los mayores índices de incidencia se obtienen cuando se pregunta si se conoce a alguna mujer en el entorno inmediato que sea víctima de malos tratos, en cuyo caso entre un 15 y un $22 \%$ de los encuestados afirman que sí conocen a alguien. Los índices más bajos se obtienen cuando se pregunta directamente si la entrevistada ha sufrido $o$ es objeto de malos tratos en el espacio familiar en el año de referencia, en cuyo caso, si los entrevistados son mujeres, las respuestas oscilan entre el 2 y el $4,2 \%$ según sea la formulación de la pregunta y el tamaño muestral (Meil, 2001). El maltrato doméstico que a través de esta encuesta se puede analizar corresponde, en consecuencia, sólo al que sufren las mujeres. Esto constituye una limitación importante, porque los estudios realizados en los Estados Unidos evidencian que la "violencia ordinaria de pareja" (Johnson y Ferraro, 2000) aplicada por las mujeres contra los hombres está tan generalizada como la aplicada por los hombres contra las mujeres. De hecho, uno de los resultados que mayor controversia ha suscitado desde los trabajos pioneros de Straus y Gelles ha sido precisamente la ausencia o no de diferencias estadísticamente significativas en función del sexo en el maltrato conyugal tal como se mide con la escala Conflict Tactics Scale desarrollada por estos autores (Kaufman y Jasinski, 1998) (Straus, 1999). El estado de la cuestión 
en la actualidad, partiendo del reconocimiento de la violencia practicada por las mujeres contra los hombres, subraya la necesidad de proceder a distinguir no sólo tipos de violencia, sino también intensidades (Johnson y Ferraro, 2000) y, sobre todo, consecuencias (Nazroo, 1999), una perpectiva que resulta difícil de satisfacer si se utiliza la técnica de las encuestas. Por otro lado, en la encuesta se recoge información igualmente sobre el causante del maltrato, que puede ser tanto el cónyuge como otros miembros de la familia, convivan o no en el mismo hogar de la entrevistada. En este trabajo se atenderá sólo al maltrato causado por el cónyuge o pareja, centrando la atención en los casos en los que hay convivencia. Nuestro objeto de estudio es, por tanto, sólo el maltrato conyugal, independientemente de cuál sea el vínculo legal entre los miembros de la pareja.

La definición de lo que es maltrato resulta también harto problemática. Dentro de la literatura científica del fenómeno existen multitud de definiciones sobre qué tipo de comportamientos deben ser considerados malos tratos. Estas definiciones suelen depender del interés de los investigadores y varían, entre otras circunstancias, según se considere el matrato conyugal o el maltrato contra los niños o la intensidad, recurrencia y métodos con que se aplica. Inicialmente se ha tendido a considerar el maltrato sólo como violencia física, definida ésta, además, únicamente desde el punto de vista de las lesiones físícas (Straus y Gelles, 1979), para ampliar posteriormente la definición e incluir también la intimidación mediante amenaza, así como el maltrato psicológico y la agresión sexual (término más amplio que el de abuso para recoger también la violación conyugal). En este trabajo, al estar basado en la "Macroencuesta sobre Violencia contra las Mujeres", se utilizarán las definiciones y operacionalización que se incluyen en dicha encuesta.

Para medir el alcance del maltrato contra las mujeres en la referida encuesta se preguntaba a las mujeres encuestadas, en primer lugar, si eran objeto en el momento de la entrevista y con qué frecuencia (frecuentemente, a veces, rara vez o nunca) de una serie de 26 comportamientos que pueden ser considerados indicadores de maltrato contra la mujer, de los cuales 13 pueden considerarse, como hacen Alberdi y Matas (2002: 130), indicadores débiles de violencia (y que reflejan sobre todo sometimiento de la mujer), mientras que los otros 13 indicadores pueden considerarse indicadores fuertes de violencia. En este artículo nos centraremos únicamente en estos últimos, que pueden agruparse, siguiendo a las mismas autoras, en cuatro dimensiones, a saber: violencia física, violencia sexual, violencia psíquica de control y violencia psíquica de desvalorización. Por otro lado, consideraremos que existe maltrato cuando uno o más de estos comportamientos se da frecuentemente o a veces, tal como se ha hecho también por parte del Instituto de la Mujer al hacer públicos los datos. Los comportamientos asociados a dichas dimensiones se encuentran recogidos en la tabla 2. Como limitaciones de dichos indicadores, sobre todo, por comparación con la Escala de Tácticas de Conflicto de Straus y Gelles, puede señalarse que la violencia 
física queda recogida de una forma muy poco específica, quedando sin poderse aislar lo que en dicha escala se denomina agresión física grave (patadas, puñetazos, golpes contra la pared, utilización de armas, entre otros), así como tampoco se recoge información sobre la dimensión de las lesiones, ya sean éstas graves (como pérdida del conocimiento, fracturas, lesiones que necesitan tratamiento médico, entre otras) o menos graves (magulladuras, dolores, etc.). De hecho, hay grandes diferencias en la incidencia de la violencia física dependiendo de cómo se defina y de los aspectos que se consideren (Johnson y Ferraro, 2000; Straus, 1999). Respecto a la violencia sexual, el indicador utilizado resulta igualmente genérico, no pudiéndose identificar si dentro de dicha dimensión se ha dado la utilización de la fuerza física o la intimidación. Además de estos indicadores que cabe calificar como indicadores objetivos de violencia doméstica contra las mujeres, esto es, definidos a partir de comportamientos registrados, también se preguntó a las entrevistadas si, desde su punto de vista, eran objeto de maltrato, es decir, si se han sentido maltratadas en el último año o alguna vez en la vida por parte de algún miembro de su entorno.

En conjunto disponemos, por tanto, de dos grandes tipos de datos sobre la incidencia del maltrato doméstico contra las mujeres, que dan lugar a estimaciones sobre su alcance muy diferentes, siendo mucho menor la conciencia sobre la existencia de maltrato que lo que cabría denominar objetivamente como comportamiento maltratador. A efectos del análisis multivariable de su prevalencia entre los distintos tipos de relaciones de pareja y de los factores socioeconómicos de los que depende, se tratará el alcance de los distintos indicadores utilizados en términos dicotómicos, esto es, hay o no maltrato de las distintas características consideradas, si bien en el caso del maltrato físico, psíquico de control y psicológico de desvalorización podría haberse construido una escala aditiva simple que midiera no sólo la presencia, sino también la intensidad del maltrato. Se ha preferido optar por dicotomizar la variable que vamos a explicar para dotar de homogeneidad al análisis estadístico, y porque nuestro objetivo investigador se centra en determinar si las distintas dimensiones del cambio familiar contribuyen a reducir la incidencia del maltrato a la mujer. Dado el carácter dicotómico de la variable a explicar se ha utilizado la técnica estadística multivariable de la regresión logística binomial.

Para medir el alcance del cambio familiar se dipone de distintos indicadores que permiten identificar las principales dimensiones del mismo. Así, por un lado, se dispone de información sobre el tipo de vínculo entre los miembros de la pareja (matrimonial, de hecho o noviazgo), así como si se trata de una primera unión o $\mathrm{no}^{1}$. Por otro lado, para medir el grado de empowerment de las mujeres se dispone

\footnotetext{
${ }^{1}$ Aunque la pregunta se refería al número de convivencias en los últimos diez años, para la gran mayoría de la población la respuesta se refiere al conjunto de su biografia de pareja.
} 
de la información socioeconómica al uso, esto es, nivel educativo y relación con la actividad, así como información sobre quién aporta más ingresos al hogar. A partir de estos datos y de los correspondientes al cónyuge pueden calcularse las diferencias de ingresos y de nivel de estudios entre los miembros de la pareja, así como el cambio en los roles de género entre los cónyuges, al menos en lo que se refiere a la incorporación de la mujer al mercado de trabajo.

Como factores predictivos del modelo se han incluido también la relación con la actividad del varón y su nivel de estudios, al ser las variables socioeconómicas (bajo nivel de ingresos, bajo nivel educativo y desempleo o trabajo irregular) factores estrechamente asociados con la violencia doméstica, si bien tampoco cabe considerarlos como causas suficientes de la misma (Kaufman y Jasinski, 1998: 27). Junto a estos factores predictivos se han introducido como variables de control el tamaño del lugar de residencia y la edad, así como el número de hijos. No obstante, el análisis no se plantea en términos de contrastación de las distintas teorías explicativas de la violencia conyugal de género, sino que ante todo se trata de identificar si el cambio familiar está asociado con una menor incidencia de la violencia conyugal de género. No se ha incluido el nivel de ingresos del hogar, puesto que si bien se recoge en la encuesta, esta variable adolece en ésta, como en todas las encuestas, de una elevada tasa de no respuesta. Su inclusión habría elevado el número de casos perdidos para el análisis por falta de información desde el 1,7 al 8,9\%, sin mejorar en nada ni el nivel de sensitividad, ni el de especificidad del modelo, así como tampoco significativamente las demás medidas de bondad del ajuste, al tiempo que hubiera alterado los estimadores de algunas de las variables relevantes del modelo.

\section{EL ALCANCE DEL MALTRATO EN EL SENO DE LA PAREJA}

En este apartado se van a presentar los resultados de dicha encuesta sobre el maltrato en el seno de las relaciones de pareja (matrimonio, cohabitación y pareja sin convivencia) antes de abordar propiamente las cuestiones planteadas en la presentación del problema, pues es una dimensión que apenas ha sido abordada en la explotación de los resultados de la Macroencuesta que se ha hecho en otras publicaciones (Alberdi y Matas, 2002). En las tablas 1 y 2 se ha recogido el alcance del maltrato según los distintos indicadores disponibles atendiendo al tipo de vínculo de pareja, así como a la relación con la actividad de la mujer. Como puede observarse, la frecuencia del maltrato objetivo es alrededor de seis veces superior a la conciencia de maltrato, incluso después de haber despertado la sensibilidad de la entrevistada al preguntar sobre si es objeto de 32 tipos de comportamientos distintos susceptibles de ser considerados maltrato. La razón de esta gran discrepancia hay que buscarla, creemos, por un lado, en el ámbito metodológico y, por otro, en razones sociales y psicológicas. Por lo que se refiere 
RIS

REVISTA INTERNACIONAL DE SOCIOLOCIA

No 37, ENERO-ABRIL, 2004

GERARDO MELL LANDWERLIN

a la dimensión metodológica, cuantos más indicadores (léase comportamientos individualizados) se consideren mayor será la proporción de encuestados englobados bajo la categoría de referencia. Así, si en lugar de considerar los 13 indicadores fuertes de maltrato, se consideran los 26 incluidos en el cuestionario, la proporción de mujeres objetivamente maltratadas pasa del 12 al $24 \%$, y si en lugar de considerar varios ítems por dimensión "fuerte" de maltrato sólo consideramos uno, la proporción se reduce del 12 al 5\% según sean los ítems que se

Tabla 1.

El maltrato a la mujer por su cónyuge o pareja según distintas definiciones del maltrato, situación de convivencia y relación con la actividad de la mujer.

Convivientes

\begin{tabular}{|c|c|c|c|c|c|}
\hline \multicolumn{6}{|c|}{$\begin{array}{c}\text { Convivientes } \\
\text { (matrimonial o de hecho) }\end{array}$} \\
\hline & Ocupada & Parada & No activa & $\begin{array}{c}\text { Tiene } \\
\text { pareja } \\
\text { pero no } \\
\text { convive }\end{array}$ & $\begin{array}{c}\text { Total } \\
\text { mujeres } \\
\text { con } \\
\text { pareja }\end{array}$ \\
\hline \multicolumn{6}{|c|}{$\begin{array}{l}\text { Porcentaje de mujeres maltratadas por cónyuge o pareja según definición objetiva* (frecuente- } \\
\text { mente o a veces) en el momento de la entrevista }\end{array}$} \\
\hline TOTAL & 10,6 & 13,7 & 14,6 & 4,0 & 11,8 \\
\hline Maltrato físico & 1,5 & 3,8 & 2,8 & 0,6 & 1,7 \\
\hline Maltrato sexual & 4,1 & 5,7 & 5,8 & 1,5 & 4,7 \\
\hline $\begin{array}{l}\text { Maltrato psíquico de } \\
\text { control }\end{array}$ & 2,8 & 4,2 & 3,6 & 1,2 & 2,3 \\
\hline $\begin{array}{l}\text { Maltrato psíquico de } \\
\text { desvalorización }\end{array}$ & 7,1 & 9,3 & 9,3 & 2,0 & 5,6 \\
\hline \multicolumn{6}{|c|}{ Porcentaje de mujeres maltratadas por cónyuge o pareja según percepción subjetiva** } \\
\hline Durante el último año & 2,0 & 3,7 & 2,5 & 1,2 & 1,9 \\
\hline Alguna vez en la vida & 3,5 & 4,9 & 3,8 & 2,2 & 3,8 \\
\hline
\end{tabular}

* La definición objetiva del maltrato se hace a partir de las respuestas positivas (frecuentemente a veces) dadas a las preguntas contenidas en la tabla 2.

** La definición subjetiva corresponde a las mujeres que han contestado afirmativamente a la pregunta "Durante el último año/ alguna vez en la vida, ¿en alguna ocasión ha sufrido alguna situación por la que Usted se haya considerado maltratada por algún familiar, por su novio o por alguna persona de las que conviven con Usted?

Fuente: Elaboración propia sobre microdatos de Instituto de la Mujer, Encuesta sobre violencia doméstica a las mujeres, Madrid, 1999 
Tabla 2.

Proporción de mujeres en pareja de todas las edades que declaran ser objeto "en la actualidad" de las referidas circunstancias (frecuentemente o a veces) por parte de su cónyuge o pareja y que definen una situación de maltrato.

\begin{tabular}{|c|c|c|c|c|}
\hline \multicolumn{5}{|c|}{$\begin{array}{l}\text { Convivientes (matrimonial o de hecho) } \\
\text { Total }\end{array}$} \\
\hline & Ocupada & Desempleada & $\begin{array}{c}\text { No } \\
\text { activa }\end{array}$ & $\begin{array}{l}\text { Mujeres } \\
\text { conviven con } \\
\text { cónyuge o } \\
\text { pareja }\end{array}$ \\
\hline \multicolumn{5}{|l|}{ VIOLENCIA FÍSICA } \\
\hline Le insulta o amenza & 1,1 & 2,0 & 1,9 & 1,5 \\
\hline En ciertas ocasiones le produce miedo & 0,9 & 2,2 & 1,4 & 1,2 \\
\hline $\begin{array}{l}\text { Cuando se enfada llega a empujar o } \\
\text { golpear }\end{array}$ & 0,5 & 1,8 & 0,9 & 0,8 \\
\hline \multicolumn{5}{|l|}{ VIOLENCIA SEXUAL } \\
\hline $\begin{array}{l}\text { Insiste en tener relaciones sexuales } \\
\text { aunque sepa que Usted no tiene ganas }\end{array}$ & 4,1 & 5,7 & 5,8 & 4,7 \\
\hline \multicolumn{5}{|c|}{ VIOLENCIA PSÍQUICA DE CONTROL } \\
\hline $\begin{array}{l}\text { Le impide ver a la familia o tener rela- } \\
\text { ciones con amigos, vecinos, etc. }\end{array}$ & 1,7 & 2,4 & 1,3 & 1,4 \\
\hline $\begin{array}{l}\text { Le quita el dinero que Usted gana o } \\
\text { no le da lo suficiente que necesita para } \\
\text { mantenerse }\end{array}$ & 0,4 & 1,3 & 0,5 & 0,5 \\
\hline $\begin{array}{l}\text { Decide las cosas que Usted puede o } \\
\text { no hacer }\end{array}$ & 1,5 & 2,4 & 2,6 & 2,0 \\
\hline \multicolumn{5}{|c|}{ VIOLENCIA PSÍQUICA DE DESVALORIZACIÓN } \\
\hline $\begin{array}{l}\text { No tiene en cuenta las necesidades de } \\
\text { Usted (le deja el peor sitio de la casa, } \\
\text { lo peor de la comida, etc.) }\end{array}$ & 2,1 & 2,2 & 2,1 & 1,9 \\
\hline $\begin{array}{l}\text { Le dice que todas las cosas que hace } \\
\text { están mal, que es torpe }\end{array}$ & 1,8 & 2,9 & 2,5 & 2,1 \\
\hline $\begin{array}{l}\text { Ironiza o no valora sus creencias (ir a la } \\
\text { Iglesia, votar a algún partido, pertene- } \\
\text { cer a alguna organización, etc.) }\end{array}$ & 1,8 & 3,1 & 1,7 & 1,6 \\
\hline No valora el trabajo que realiza & 3,7 & 6,8 & 6,2 & 4,7 \\
\hline $\begin{array}{l}\text { Delante de sus hijos (si los tiene) dice } \\
\text { cosas para no dejarle en buen lugar }\end{array}$ & 2,2 & 3,7 & 3,1 & 2,4 \\
\hline $\begin{array}{l}\text { Tamaño de la submuestra (valores } \\
\text { ponderados) }\end{array}$ & 2.820 & 546 & 5.795 & 11.007 \\
\hline
\end{tabular}

Leyenda: La diferencia de cada número hasta 100 es la proporción de mujeres que no padecen la situación que cada ítem describe.

Fuente: Elaboración propia sobre microdatos de Instituto de la Mujer, Encuesta sobre violencia doméstica a las mujeres, Madrid, 1999. 
seleccionen dentro de cada dimensión, sin que por ello haya que concluir que se manipulan los datos. Por otro lado, las líneas de conflicto verbal dentro de la pareja llevan a cristalizar modos de relación y de agresiones verbales que con el tiempo se perciben como "normales" por sus protagonistas y no como maltrato. Lo mismo sucede en muchos casos con las agresiones físicas, circunstancia facilitada además en buena medida por las normas sociales que sancionan positivamente determinadas formas de maltrato (Dobash y Dobash, 1992; Lorente, 2001). Así, sólo un $16 \%$ de las mujeres maltratadas objetivamente por su cónyuge o pareja se siente maltratada, si bien hay también un $15 \%$ de mujeres que se sienten maltratadas a pesar de no señalar ser víctima con frecuencia o a veces de alguna de las agresiones recogidas en la tabla 2.

El tipo de maltrato objetivo más frecuente es el maltrato psíquico y particularmente el de desvalorización, siendo lo más frecuente la desvalorización del trabajo realizado por la mujer, de sus capacidades como persona o como esposa/ pareja, así como, eventualmente, de sus creencias. Esta desvalorización se da con mayor frecuencia hacia mujeres que son amas de casa o están desempleadas, aunque también cabe encontrarla entre las mujeres ocupadas e incluso cuando ganan más que sus cónyuges, pues no es sino manifestación de una voluntad de superioridad por parte del varón por su condición de tal y por encima de cualquier otra circunstancia y consideración: es voluntad de dominación. La descalificación mutua es, de hecho, una de las formas típicas que adopta el conflicto dentro de las parejas y no es, además, de carácter unidireccional. La violencia sexual por parte de la pareja o del cónyuge aparece como la segunda dimensión más frecuente de maltrato, incluso más extendida que el control de los movimientos de la mujer. Al respecto hay que destacar, no obstante, que la redacción de la pregunta no resulta demasiado afortunada al incluir el calificativo de "insistir" en lugar de una formulación más dura como sería la de obligar, si bien podría considerarse, tal como lo hacen Alberdi y Matas (2002: 130), que en el contexto en el que se formula resulta satisfactoria para recoger situaciones de maltrato sexual en el seno de la pareja. La violencia física, tal como se recoge en esta encuesta, es la dimensión menos frecuente, lo que no significa que carezca de presencia social, pues un punto porcentual representa en términos absolutos ni más ni menos que 168.000 mujeres maltratadas fisicamente.

La correlación que se da entre las distintas dimensiones del maltrato es más bien modesta, pues se cifra en 0,45 entre la violencia de control y la violencia de desvalorización, en 0,56 entre violencia física y violencia de desvalorización y en 0,46 entre violencia física y violencia de control. La mayoría de las mujeres maltratadas (58\%) es objeto de uno de los 13 comportamientos que definen las distintas dimensiones consideradas, mientras que un $15 \%$ dicen ser víctimas con frecuencia o a veces de 3 o más de dichos comportamientos, proporción esta última que representa un $1,6 \%$ del total de las mujeres encuestadas, lo que en términos absolutos supone un total de 270.000 mujeres. 
Para la gran mayoría de las mujeres estas situaciones de maltrato se producen de forma continuada en el tiempo, de forma que en tres de cada cuatro casos se dan desde hace más de 5 años, no habiendo diferencias sustanciales según el tipo de maltrato, pues los porcentajes varían entre el $71 \%$, en el caso del maltrato sexual hasta el $77 \%$, en el caso del maltrato psíquico de desvalorización. Las mayores diferencias se producen en función del tipo de vínculo de pareja, que no hacen sino reflejar la distinta duración media de las relaciones de pareja; no obstante, queda abierta la pregunta de si en los contextos no institucionalizados de pareja el nivel de tolerancia hacia el maltrato es menor y se disuelven, por tanto, antes que los matrimonios, una cuestión que no puede abordarse con los datos proporcionados por la encuesta.

Si atendemos a la percepción subjetiva, el maltrato psicológico es el más frecuentemente citado por quienes afirman haberse sentido maltratadas por su pareja (concretamente un $89 \%$ ) lo que supone que un $3 \%$ de las mujeres con pareja (matrimonial o de hecho) se sienten maltratadas psicológicamente. La segunda dimensión más extendida es el maltrato físico, que afecta al $0,7 \%$ de las mujeres con pareja, una proporción casi idéntica a la que se obtiene con el

Tabla 3.

Dimensiones de la percepción subjetiva de maltrato por parte del entorno familiar (cónyuge/pareja, hijos, padres, etc.). En porcentajes sobre el total de mujeres que han respondido haberse sentido maltratada durante el año anterior a la entrevista.

\begin{tabular}{lccccc}
\hline & \multicolumn{5}{c}{ Tiene pareja } \\
& Matrimonios & $\begin{array}{c}\text { Uniones de } \\
\text { hecho }\end{array}$ & $\begin{array}{c}\text { pero no } \\
\text { convive }\end{array}$ & Sin pareja & Total \\
\hline Psicológicamente & 85,6 & 92,1 & 87,8 & 89,4 & 87,9 \\
Físicamente & 19,0 & 36,4 & 21,1 & 28,2 & 24,3 \\
Sexualmente & 1,0 & 6,3 & - & 2,8 & 2,2 \\
Espiritualmente & - & - & - & - & 0,5 \\
Económicamente & 1,6 & - & - & 5,0 & 3,0 \\
Estructuralmente & 1,5 & - & - & 1,7 & 1,7 \\
Ns/nc & 2,0 & - & - & - & 1,4 \\
Otras & 5,9 & - & 7,1 & 4,5 & 5,3 \\
Total & 117 & 135 & 106 & 132 & 127 \\
Total submuestra & 348 & 57 & 110 & 381 & 895 \\
\hline
\end{tabular}

Nota: La suma es superior a 100 por admitirse respuesta múltiple. No se pueden identificar las caracteristicas del maltrato por parte del cónyuge por haber respondido sólo 46 mujeres de las 292 entrevistadas que se sentían maltratadas por el cónyuge/pareja.

Fuente: Elaboración propia sobre microdatos de Instituto de la Mujer, Encuesta sobre violencia doméstica a las mujeres, Madrid, 1999. 
indicador objetivo, si bien ninguna de estas mujeres que se sienten maltratadas físicamente declararon por otro lado que "cuando se enfada (su pareja) llega a empujar o golpear". La percepción subjetiva de ser objeto de agresión sexual en el seno de la pareja se eleva al $0,1 \%$ de las mujeres entrevistadas, lo que indica que en la inmensa mayoría de los casos en los que hay agresión sexual en el seno de la pareja, aunque sea contra la voluntad de la mujer, ésta termina aceptándolo como propio de la relación de pareja, y no sólo en las relaciones matrimoniales, sino también en aquéllas que no están institucionalizadas.

\section{CAMBIO FAMILIAR Y MALTRATO CONYUGAL}

Una vez presentados los datos agregados pasamos a discutir los resultados del análisis multivariable para comprobar si el cambio en los roles de género y el consecuente cambio familiar comportan una menor incidencia del maltrato hacia la mujer, una vez controlados los efectos de las demás variables relevantes. En la tabla 4 se han recogido las estimaciones de la razón de probabilidades de maltrato objetivo y de maltrato subjetivo, esto es, por un lado, la razón estimada entre la probabilidad de que una mujer sea maltratada por su cónyuge en su definición objetiva y entre la probabilidad de que no lo sea y, por otro lado, la razón estimada entre la probabilidad de que una mujer se sienta maltratada por su cónyuge y entre la probabilidad de que no se sienta maltratada, para cada uno de los valores que toman las variables independientes. Estas razones de probabilidades pueden interpretarse en términos de riesgo de maltrato. Como es habitual, representamos con asteriscos los distintos niveles de significación, de forma que cuando no hay asterisco alguno, las diferencias en las razones de probabilidades estimadas (el riesgo estimado de maltrato) entre la categoría de referencia y la categoría de comparación no resultan estadísticamente significativas. Por otro lado, valores mayores que 1 representan un mayor riesgo de maltrato por comparación.con la categoría de referencia y valores por debajo de 1 representan un menor riesgo de maltrato.

\section{Cambio en los roles de género}

En las últimas décadas se ha producido una incorporación masiva de las mujeres al mercado de trabajo. Esta incorporación de la mujer al trabajo remunerado ha posibilitado, por un lado, su acceso a recursos monetarios que son fundamentales para la supervivencia en una economía de mercado y, por otro, le permite lograr un mayor estatus social, dada la importancia simbólica atribuida al trabajo extradoméstico en la estratificación social de las sociedades modernas, en las que se ha tendido a devaluar cada vez más el trabajo no remunerado en el espacio doméstico. La obtención de estos recursos se ha traducido en la ganancia 
de cuotas de poder para poder configurar sus proyectos de vida con una mayor independencia y para negociar desde una posición más fuerte el contenido de los roles en el seno de la pareja, así como para concluir una relación de pareja si ésta resulta insatisfactoria. Como consecuencia de ello sería de esperar una menor frecuencia de maltrato, algo que parece verse respaldado por la desagregación de los índices de violencia en función del estatus laboral de la mujer, tal como puede observarse en las tablas 1 y 2 .

Los resultados del análisis de regresión logística recogidos en las tablas 4 y 5 en los que se analiza dicha relación, controlada la influencia de otros factores relevantes como son -entre otros- el número de hijos (que está condicionado a su vez también por el status laboral de la mujer), evidencian que la menor incidencia del maltrato entre las mujeres ocupadas y, por tanto, con ingresos propios que se observa en las tablas 1 y 2 resulta mucho menos clara de lo que a primera vista puede deducirse y, sobre todo, más paradójica. Así, si centramos la atención en la definición objetiva de maltrato, podemos observar que el riesgo de maltrato entre las mujeres que son amas de casa no puede afirmarse que sea superior al de las mujeres ocupadas si asumimos un nivel de significación convencional del 5\%. Sólo si ampliamos el riesgo de cometer un error al afirmar que hay diferencias cuando de hecho no las hay podemos afirmar que las mujeres ocupadas tienen menor riesgo de maltrato que las amas de casa. Pero incluso en este caso no puede afirmarse que las diferencias sean muy grandes, pues las segundas tendrían "sólo" un $20 \%$ más de riesgo que las primeras (lo que comparado con más del $200 \%$ que tienen las madres de familia numerosa frente a las que no tienen hijos es ciertamente muy bajo). Donde sí se presentan diferencias estadísticamente significativas es, por el contrario, en el caso de las mujeres desempleadas, que tienen, además, un riesgo mayor que las amas de casa de ser maltratadas en comparación con las mujeres ocupadas (la razón de odds es un 30\% superior frente al citado $20 \%$ ). Es decir, que las mujeres que mayor riesgo tienen de ser maltratadas (en su definición objetiva) no son las mujeres que viven en el marco de un modelo tradicional de división de los roles, sino aquéllas que han abandonado el modelo tradicional y no han encontrado empleo, y que viven, por tanto, en un contexto marcado por unas expectativas de ingresos más elevadas, que se ven, sin embargo, frustradas por la situación del mercado de trabajo. Esta sorprendente pauta se da no sólo en el maltrato de desvalorización, sino en todas las dimensiones del maltrato consideradas salvo en el maltrato sexual, siendo el riesgo de maltrato físico especialmente elevado. El riesgo de ser agredida, amenazada o de temer por la integridad física es casi tres veces superior entre las mujeres desempleadas que entre las mujeres ocupadas. $\mathrm{O}$ expresado desde otra perspectiva, en el caso de que haya maltrato asociado a una situación de desempleo, la probabilidad de que este maltrato sea físico es mucho mayor que lo sea de tipo psíquico. 
Tabla 4.

Modelos de regresión logistica de los factores explicativos del maltrato por el cónyuge/pareja con el que convive.

\begin{tabular}{|c|c|c|}
\hline & Maltrato objetivo & $\begin{array}{c}\text { Percepción subjetiva } \\
\text { de maltrato }\end{array}$ \\
\hline $\begin{array}{l}\text { Número de hijos: } \\
\text { No tiene hijos } \\
\text { Un hijo } \\
\text { Dos hijos } \\
\text { Tres hijos } \\
\text { Cuatro o más hijos } \\
\end{array}$ & $\begin{array}{l}1 \\
1,4 * \\
1,6 * * * \\
2,1 * * * \\
2,2 * * * \\
\end{array}$ & $\begin{array}{l}1 \\
4,2^{* * *} \\
4,3^{* * *} \\
7,6^{* * *} \\
7,3^{* * *}\end{array}$ \\
\hline $\begin{array}{l}\text { Tipo de relación de pareja: } \\
\text { Matrimonial } \\
\text { De hecho }\end{array}$ & $\begin{array}{l}1 \\
1,3\end{array}$ & $\begin{array}{l}1 \\
2,9 * * *\end{array}$ \\
\hline $\begin{array}{l}\text { Diferencia en el nivel educativo de los } \\
\text { miembros de la pareja: } \\
\text { Homogamia } \\
\text { Hipergamia } \\
\text { Hipogamia }\end{array}$ & $\begin{array}{l}1 \\
1,1 \\
1,2 *\end{array}$ & $\begin{array}{l}1 \\
2,0^{* * *} \\
1,2\end{array}$ \\
\hline Tamaño del municipio & 1,04 & $1,1^{*}$ \\
\hline $\begin{array}{l}\text { Número de convivencias en los últimos } \\
10 \text { años: } \\
\text { Una } \\
\text { Más de una }\end{array}$ & $\begin{array}{l}1 \\
1,4+\end{array}$ & $\begin{array}{l}1 \\
1,3\end{array}$ \\
\hline Edad & 0,996 & 1 \\
\hline $\begin{array}{l}\text { Relación con la actividad de las mujeres: } \\
\text { Ocupada } \\
\text { Parada } \\
\text { No trabaja fuera del hogar } \\
\end{array}$ & $\begin{array}{l}1 \\
1,3 * \\
1,2+\end{array}$ & $\begin{array}{l}1 \\
1,7^{*} \\
1,1\end{array}$ \\
\hline $\begin{array}{l}\text { Relación con la actividad del cónyuge: } \\
\text { Trabaja a tiempo completo } \\
\text { Trabaja a tiempo parcial } \\
\text { No trabaja }\end{array}$ & $\begin{array}{l}1 \\
1,3 \\
1,1 \\
\end{array}$ & $\begin{array}{l}1 \\
1,2 \\
1,1\end{array}$ \\
\hline $\begin{array}{l}\text { Nivel de estudios del cónyuge: } \\
\text { Primarios o menos } \\
\text { Secundarios } \\
\text { Universitarios } \\
\end{array}$ & $\begin{array}{l}1 \\
0,7 * * * \\
0,6 * * *\end{array}$ & $\begin{array}{l}1 \\
1,0 \\
0,7\end{array}$ \\
\hline $\begin{array}{l}\text { ¿Quién gana más en el hogar? } \\
\text { El cónyuge/pareja (varón) } \\
\text { La mujer u otra persona }\end{array}$ & $\begin{array}{l}1 \\
0,9\end{array}$ & $\begin{array}{l}1 \\
1,0\end{array}$ \\
\hline $\begin{array}{l}\text { Sensitividad: } \% \text { predicción } y=1 \\
\text { Especificidad: \% total de aciertos }\end{array}$ & $\begin{array}{l}0 \\
87\end{array}$ & $\begin{array}{l}0 \\
98\end{array}$ \\
\hline
\end{tabular}

Fuente: Elaboración propia sobre microdatos de Instituto de la Mujer, Encuesta sobre violencia doméstica a las mujeres, Madrid, 1999 
Tabla 5.

Modelos de regresión logística de los factores explicativos del maltrato por el cónyuge/pareja con el que convive según su definición objetiva.

\begin{tabular}{|c|c|c|c|c|}
\hline & $\begin{array}{c}\text { Violencia } \\
\text { fisica }\end{array}$ & $\begin{array}{c}\text { Violencia } \\
\text { sexual }\end{array}$ & $\begin{array}{l}\text { Violencia } \\
\text { de control }\end{array}$ & $\begin{array}{l}\text { Violencia } \\
\text { psíquica }\end{array}$ \\
\hline $\begin{array}{l}\text { Número de hijos: } \\
\text { No tiene hijos } \\
\text { Un hijo } \\
\text { Dos hijos } \\
\text { Tres hijos } \\
\text { Cuatro o más hijos }\end{array}$ & $\begin{array}{l}1 \\
1,6 \\
2,1^{*} \\
2,9^{* *} \\
2,8^{* *} \\
\end{array}$ & $\begin{array}{l}1 \\
1,8^{*} \\
2,1^{* *} \\
3,3^{* * *} \\
2,8^{* *} \\
\end{array}$ & $\begin{array}{l}1 \\
2,0 * * \\
2,1 * * \\
2,4 * * \\
2,9 * * *\end{array}$ & $\begin{array}{l}1 \\
1,8 * * \\
2,0 * * * \\
2,6 * * * \\
2,7 * * *\end{array}$ \\
\hline $\begin{array}{l}\text { Tipo de relación de pareja: } \\
\text { Matrimonial } \\
\text { De hecho }\end{array}$ & $\begin{array}{l}1 \\
1,2\end{array}$ & $\begin{array}{l}1 \\
1,2\end{array}$ & $\begin{array}{l}1 \\
1,5\end{array}$ & $\begin{array}{l}1 \\
1,5 *\end{array}$ \\
\hline $\begin{array}{l}\text { Diferencia en el nivel educativo } \\
\text { de los miembros de la pareja: } \\
\text { Homogamia } \\
\text { Hipergamia } \\
\text { Hipogamia }\end{array}$ & $\begin{array}{l}1 \\
1,6^{*} \\
1,4^{*}\end{array}$ & $\begin{array}{l}1 \\
0,9 \\
1,0\end{array}$ & $\begin{array}{l}1 \\
1,0 \\
1,1 \\
\end{array}$ & $\begin{array}{l}1 \\
1,3 * \\
1,2+\end{array}$ \\
\hline Tamaño del municipio & 1,09 & 1,06 & 1,04 & $1,1^{* *}$ \\
\hline $\begin{array}{l}\text { Número de convivencias en los } \\
\text { últimos } 10 \text { años: } \\
\text { Una } \\
\text { Más de una }\end{array}$ & $\begin{array}{l}1 \\
1,1 \\
\end{array}$ & $\begin{array}{l}1 \\
2,6 * * \\
\end{array}$ & $\begin{array}{l}1 \\
2,8 * * * \\
\end{array}$ & $\begin{array}{l}1 \\
1,3 \\
\end{array}$ \\
\hline Edad & 0,998 & 0,989 & $0,984 *$ & 0,997 \\
\hline $\begin{array}{l}\text { Relación con la actividad de } \\
\text { las mujeres: } \\
\text { Ocupada } \\
\text { Parada } \\
\text { No trabaja fuera del hogar }\end{array}$ & $\begin{array}{l}1 \\
2,7 * * \\
1,4\end{array}$ & $\begin{array}{l}1 \\
0,9 \\
0,9\end{array}$ & $\begin{array}{l}1 \\
1,5+ \\
1,2\end{array}$ & $\begin{array}{l}1 \\
1,3+ \\
1,1 \\
\end{array}$ \\
\hline $\begin{array}{l}\text { Relación con la actividad del } \\
\text { cónyuge: } \\
\text { Trabaja a tiempo completo } \\
\text { Trabaja a tiempo parcial } \\
\text { No trabaja }\end{array}$ & $\begin{array}{l}1 \\
1,3 \\
1,8^{* *}\end{array}$ & $\begin{array}{l}1 \\
1,9 * \\
1,5+\end{array}$ & $\begin{array}{l}1 \\
1,4 \\
2,0 * * *\end{array}$ & $\begin{array}{l}1 \\
1,6 * * \\
1,2\end{array}$ \\
\hline $\begin{array}{l}\text { Nivel de estudios del cónyuge: } \\
\text { Primarios o menos } \\
\text { Secundarios } \\
\text { Universitarios } \\
\end{array}$ & $\begin{array}{l}1 \\
0,9 \\
0,6^{*} \\
\end{array}$ & $\begin{array}{l}1 \\
0,7+ \\
0,6 \\
\end{array}$ & $\begin{array}{l}1 \\
1,1 \\
0,9 \\
\end{array}$ & $\begin{array}{l}1 \\
0,7 * * \\
0,7 * *\end{array}$ \\
\hline $\begin{array}{l}\text { ¿Quién gana más en el hogar? } \\
\text { El cónyuge/pareja (varón) } \\
\text { La mujer u otra persona }\end{array}$ & $\begin{array}{l}1 \\
0,7^{*}\end{array}$ & $\begin{array}{l}1 \\
0,7^{* *}\end{array}$ & $\begin{array}{l}1 \\
0,9\end{array}$ & $\begin{array}{l}1 \\
0,9\end{array}$ \\
\hline $\begin{array}{l}\text { Sensitividad: \% predicción } y=1 \\
\text { Especificidad: \% total de aciertos }\end{array}$ & $\begin{array}{l}0 \\
98 \\
\end{array}$ & $\begin{array}{l}0 \\
97 \\
\end{array}$ & $\begin{array}{l}0 \\
97\end{array}$ & $\begin{array}{l}0 \\
91 \\
\end{array}$ \\
\hline
\end{tabular}

Fuente: Elaboración propia sobre microdatos de Instituto de la Mujer, Encuesta sobre violencia doméstica a las mujeres, Madrid, 1999 
Si en lugar de considerar el maltrato objetivo, consideramos la percepción subjetiva de maltrato, que podemos suponer que puede ser mayor entre las mujeres que ya no se comportan con arreglo al modelo tradicional de roles de género, puede observarse, no obstante, exactamente el mismo patrón: el abandono del modelo tradicional de definición de los roles no reduce el riesgo de unas relaciones conyugales que se perciben como propias de maltrato, antes bien, al contrario, aumenta dicho riesgo cuando la mujer no consigue estabilizar su relación laboral y se encuentra desempleada. En este sentido, el cambio familiar en su dimensión de cambio en la definición de los roles domésticos no sólo no ha comportado una reducción del riesgo de maltrato de las mujeres, tanto en su dimensión objetiva como si se mide a través de cómo se viven las relaciones conyugales, sino que habría aumentado dicho riesgo cuando las mujeres no consiguen un empleo que posibilite un mayor nivel de vida a la familia.

Si el trabajo extradoméstico como tal, esto es, el estatus que proporciona trabajar fuera de casa frente a trabajar en casa, no contribuye a reducir el riesgo de maltrato a las mujeres, el que la mujer gane más dinero que su cónyuge no está tampoco nítidamente asociado con un menor riesgo de maltrato. Así, sólo hay un menor riesgo de maltrato en las dimensiones del maltrato físico y sexual, pero no así en el maltrato psíquico, ya sea de control o de desvalorización, lo que en conjunto hace que no pueda afirmarse con suficiente fundamento empírico que las mujeres que ganan más que sus cónyuges tengan un menor riesgo de maltrato objetivo. Y tampoco si se considera la percepción subjetiva de maltrato. Por tanto, los logros de las mujeres en el mercado de trabajo (y, por tanto, en la esfera pública) no están nítida y contundentemente asociados con la superación del maltrato dentro de la pareja, y en este sentido no hay fundamento para esperar una reducción del maltrato conyugal por el mero hecho de que las mujeres trabajen y ganen independencia económica y estatus respecto a sus cónyuges o parejas. Además de que las mujeres ganen recursos de poder, es necesario que se produzca sobre todo un cambio en los valores y percepciones sociales de los varones, junto con un profundo cambio en su socialización. Los cambios estructurales no son suficientes para generar un cambio cultural que acabe con todos los restos de machismo y de patriarcado.

Los resultados sobre las diferencias educativas no confirman ni desmienten claramente esta conclusión y lo que indican, por el contrario, es que las grandes diferencias educativas entre los cónyuges están asociadas con un mayor riesgo de maltrato, un resultado que también se ha constatado para los Estados Unidos (Kaufman y Jasinski, 1998). Así, las mujeres con un menor nivel educativo que su cónyuge o pareja tienen también un mayor riesgo de maltrato físico, un mayor riesgo de maltrato de desvalorización y es mucho más probable también que se sientan subjetivamente maltratadas. Las mujeres con un mayor nivel educativo que su cónyuge o pareja no presentan un menor riesgo de maltrato; por el contrario, aunque no tienen una conciencia de mal- 
trato significativamente diferente de las mujeres homógamas, tienen un $40 \%$ mayor de riesgo de ser maltratadas físicamente por su cónyuge que éstas. La prolongación del tiempo que pasan las mujeres en el sistema educativo y el hecho de que sus resultados académicos superen los de los varones tampoco aparece claramente asociado con un menor riesgo de maltrato. Esta conclusión se ve reforzada, además, si consideramos que la edad no resulta significativa en ninguno de los casos.

\section{La desinstitucionalización del vínculo conyugal}

Si atendemos al tipo de vínculo conyugal, recogiendo sólo los casos en los que hay convivencia continuada, puede observarse que aunque la razón de probabilidades estimada del maltrato objetivo es mayor entre las mujeres que conviven de hecho que entre las mujeres casadas, estas diferencias no resultan estadísticamente significativas, por lo que no puede afirmarse que las mujeres en una unión de hecho tengan una mayor probabilidad de ser maltratadas que una mujer casada, a diferencia de los resultados obtenidos por Stets y Straus (1990) para Estados Unidos y confirmados sólo parcialmente en investigaciones posteriores (Johnson y Ferraro, 2000), o los que se obtienen de las estadísticas de asesinatos en nuestro país (Alberdi y Matas, 2000). No obstante, si se distingue entre los distintos tipos de maltrato, sí aparecen diferencias estadísticamente significativas en la dimensión de desvalorización de la mujer, siendo más probable que se dé en las uniones de hecho que en los matrimonios, si bien no en las demás dimensiones de maltrato consideradas. En conjunto, como se ha indicado, no puede afirmarse con suficiente fundamento empírico que haya mayor riesgo de maltrato en las uniones de hecho que en los matrimonios. Donde mayores y más claras diferencias se registran es en la percepción subjetiva del maltrato, donde la razón de probabilidades de maltrato estimada es tres veces mayor en las uniones de hecho que en los matrimonios.

¿Cabe concluir a partir de estos datos que en las uniones de hecho el maltrato hacia la mujer es más frecuente que en los matrimonios? No creemos que con los datos disponibles pueda fundamentarse tal conclusión. En primer lugar, es preciso considerar que el mayor riesgo de maltrato en su definición objetiva no se produce de forma consistente a lo largo de todas las dimensiones o tipos considerados; de hecho sólo aparece en una dimensión y la significatividad de las diferencias desaparece al proceder a la agregación. En segundo lugar, en las uniones de hecho hay un cuestionamiento de los modelos sociales heredados sobre la forma de organización de la vida en pareja y en consecuencia de los modelos de rol de género, por lo que la desvalorización del otro podría ser resultado de las dinámicas de conflicto generadas por este cuestionamiento de los modelos de rol, afectando probablemente no sólo a las mujeres sino también a los hombres. Por último, la mayor conciencia de maltrato por parte de las mujeres en unión de 
hecho no tiene por qué significar necesariamente que son más frecuentemente maltratadas que las mujeres casadas, sino que también puede derivar de una mayor conciencia y rechazo hacia formas de relación que las mujeres casadas pueden considerar como "normales" en la vida de pareja y, por tanto, no percibirlas como maltrato.

Lo que en cualquier caso queda firmemente establecido por estos resultados es que el cuestionamiento del matrimonio como modelo para organizar las relaciones de pareja, aunque tienda a comportar unas relaciones algo más igualitarias entre los géneros (particularmente en lo que se refiere a la participación en las tareas domésticas) (Meil, 2003), no conlleva por sí mismo un menor riesgo de maltrato. Esta conclusión se mantiene controlados también los efectos de la clase social (a través del nivel de estudios) y hasta cierto punto también de la situación de ingresos (a través de la relación con la actividad del cónyuge, así como si se incluye la variable nivel de ingresos), por lo que no puede deducirse que el riesgo de maltrato sea menor para determinados tipos de uniones de hecho que para los matrimonios en situación estrictamente comparable. Nuevamente nos encontramos, por tanto, que el cambio familiar y el cuestionamiento de los modelos tradicionales de organización de la vida doméstica no conlleva por sí mismo una reducción del riesgo de maltrato.

Otra dimensión sustantiva del cambio familiar altamente relevante en este contexto es la ruptura de pareja y la eventual formación de un nuevo proyecto de vida en común. Respecto al riesgo de maltrato derivado de la separación o del divorcio, que en la literatura se encuentra ampliamente fundamentada (Kaufman y Jasinski, 1998), esta variable no ha podido ser incluida en el análisis porque, por un lado, la definición objetiva del maltrato se recoge a partir de las características de las relaciones en el momento de la entrevista y, por otro lado, porque en la encuesta no se recogía como categoría diferenciada a la expareja. No obstante, sí se tiene información sobre la existencia de una pareja anterior, por lo que se ha incluido, como hemos señalado más arriba, en el análisis multivariable que hemos realizado. Si consideramos el indicador agregado de maltrato en su definición objetiva, es necesario asumir un riesgo de cometer un error por encima del nivel convencional del $5 \%$ para afirmar que las mujeres que inician un segundo (o sucesivo) proyecto de vida en común tienen un mayor riesgo de ser maltratadas. Ahora bien, si consideramos las distintas dimensiones que componen este indicador puede observarse que las mujeres que viven en una segunda o sucesiva unión tienen casi tres veces más riesgo de ser objeto de maltrato sexual o de maltrato psíquico de control que quienes no han roto (en los últimos 10 años) su proyecto de vida en común, pudiéndose establecer además dicha relación con un nivel de significación muy alto. La percepción subjetiva de ser objeto de maltrato no está, sin embargo, más extendida entre estas mujeres que entre quienes no han iniciado un segundo (o sucesivo) proyecto de vida en común. De estos resultados dificiles de interpretar, pues habría que 
esperar una pauta de respuesta similar a la analizada para las uniones de hecho, la única conclusión que puede establecerse con claridad es que la posibilidad de romper una relación insatisfactoria e iniciar un nuevo proyecto de vida en común no implica un menor riesgo de maltrato y que, por tanto, nuevamente el cambio familiar no está asociado con una reducción del riesgo de maltrato hacia la mujer.

\section{La reducción del tamaño de familia}

De todas las variables incluidas en el modelo explicativo, la variable más claramente relacionada con el riesgo de maltrato conyugal hacia la mujer, controladas las demás variables, es el tamaño de la familia. Aunque no de forma estrictamente lineal, a medida que aumenta el número de hijos aumenta el riesgo de maltrato, tanto en su definición objetiva como en la percepción subjetiva. Las parejas en las que menor riesgo de maltrato cabe encontrar es en las parejas sin hijos, mientras que en las familias numerosas, con 3 o más hijos, es donde mayor riesgo de maltrato cabe encontrar. Así, el riesgo de maltrato en su definición objetiva aumenta un $40 \%$ con la llegada del primer hijo y es algo más del doble cuando la mujer tiene tres o más hijos que cuando no tiene ninguno. Este mayor riesgo de maltrato conyugal asociado a la tenencia de hijos se da en todas las dimensiones consideradas, tanto en el maltrato físico y sexual, como en el psíquico de control y de desvalorización. No obstante, si en conjunto el riesgo de maltrato objetivo es mayor en las familias numerosas que en las familias reducidas o en aquéllas que no tienen hijos, hay diferencias significativas según el tipo de maltrato conyugal considerado. Así, en el caso de maltrato físico y de control las diferencias en función del tamaño de la familia no son estadísticamente significativas, siendo la única diferencia significativa la que existe entre tener o no hijos. En el caso del maltrato sexual, por el contrario, el riesgo de maltrato entre las madres de familia numerosa es un $50 \%$ superior al de las mujeres con dos hijos, y un $30 \%$ superior en el caso del maltrato de desvalorización.

Las diferencias en la percepción subjetiva abundan en la misma dirección de un mayor riesgo de maltrato asociado a la maternidad, siendo las diferencias incluso mucho más acentuadas que en el caso del maltrato objetivo. Así, el riesgo de sentirse maltratada por el cónyuge es algo más de cuatro veces mayor cuando se tiene uno o dos hijos que cuando no se tiene ninguno y más de siete veces cuando se tienen tres o más. En este sentido, la materialización de un proyecto familiar por la pareja con la tenencia y educación de hijos no comporta una reducción del riesgo de ser maltratada para las mujeres; por el contrario, las hace potencialmente más vulnerables, sin que de ello se pueda deducir que los hijos son los causantes indirectos del maltrato conyugal. 
RIS

REVISTA INTERNACIONAL DE SOCIOLOCIA

№ 37, ENERO-ABRIL, 2004

GERARDO MELL LANDWERLIN

\section{CONCLUSIONES}

La vida social en general y la vida familiar en particular está sujeta a un profundo proceso de cambio que ha alterado profundamente la definición social de los roles de género dentro y fuera del espacio doméstico. El desarrollo de una cultura más igualitaria en las relaciones entre los géneros, la masiva y prolongada permanencia de las mujeres en el sistema educativo y su posterior incorporación al mercado de trabajo, mayormente ya no interrumpida por la maternidad, han supuesto un aumento sustancial de sus recursos de poder y de las bases sociales y económicas para su independencia. Este incremento de los recursos de poder ha comportado un cuestionamiento de las tradicionales relaciones de poder en el seno de la pareja y una mayor capacidad para definir y redefinir aspectos básicos de las relaciones entre los géneros con el fin de lograr un orden familiar más simétrico y satisfactorio para todos los actores sociales implicados. Más en concreto, como consecuencia de estos profundos cambios sería de esperar que este aumento de poder se tradujera en una disminución de los malos tratos en el seno de la pareja y, en particular, del maltrato hacia la mujer. Los análisis que hemos realizado a partir de la macroencuesta sobre violencia doméstica, no permiten sin embargo, augurar una disminución de los malos tratos hacia la mujer por parte de su cónyuge o pareja como consecuencia "automática" de este aumento de las bases de poder de las mujeres. La única dimensión del cambio familiar de las que hemos podido analizar que está clara y fuertemente asociada con un menor riesgo de maltrato es el control de la fecundidad y la correspondiente reducción del tamaño de la familia. La incorporación de la mujer al mercado de trabajo, por el contrario, no está claramente asociada a un menor riesgo de maltrato conyugal, al menos no con todos los tipos de maltrato considerados, incluso cuando ello se traduce en unos ingresos iguales o superiores a los de su cónyuge o pareja. Igualmente, la mejora de los niveles educativos y la formación de parejas en las que la mujer tiene un mayor nivel de formación que su cónyuge tampoco se traduce en un menor riesgo de maltrato. A mayor nivel educativo del varón, sin embargo, disminuye el riesgo de maltrato de la mujer, lo que sugiere, si se interpreta como una variable representativa de la ideología de rol, que avances significativos en la reducción del riesgo de maltrato pueden lograrse (entre otras vías) si se modifican las representaciones sociales sobre los modelos de rol de género entre los varones. No obstante, un conocimiento más completo de la realidad de los malos tratos en el ámbito familiar requiere de una perspectiva complementaria que incluya también a los varones como potenciales víctimas, $\mathrm{y}$ de escalas de medición alternativas y complementarias a las utilizadas en la encuesta que hemos analizado. 


\section{REFERENCIAS BIBLIOGRÁFICAS}

ALBERDI, I y N. MATAS (2002), La violencia doméstica, Barcelona, Fundación La Caixa.

DOBASH, , R.E. y R.P. DOBASH (1992), Women, Violence and Social Change, Nueva York, Routledge.

JOHNSON, M.P. y K.J. FERRARO (2000), "Research on Domestic Violence in the 1990s: Making Distinctions", Journal of Marriage and the Family, $\mathrm{n}^{\circ}$ 62, 4, pp. 948-963.

GELLES, R. y M. STRAUS (1979), "Determinants of violence in the Family: Towards a theoretical integration", en W.R. BURR. R. HILL, F.I. NYE y I.L. REISS (eds), Contemporary Theories about the Family, The Free Press, Nueva York.

GELLES, R. J. y CL.P. CORNELL (1990), Intimate Violence in Families, Sage, Newbury Park, 2. ed.

KAUFMAN KANTOR, G. y J. L. JASINSKI (1998), "Dynamics and Risk Factors in Partner Violence", en JASINSKI, J.L. y L.M. WILLIAMS (eds), Partner Violence. A Comprehensive Review of 20 Years Research, Sage, Thousand Oaks, pp. 1-43.

LORENTE, M. (2001), Mi marido me pega lo normal. Agresión a la mujer, realidades y mitos, Barcelona, Edit. Ares y Mares.

MEIL, G. (2001), "La violencia doméstica en el contexto del cambio familiar. Una perspectiva sociológica", Cuadernos de Derecho Judicial, n 5, 2001, pp. 77-99.

(2003), Las uniones de hecho en España, CIS, col. monografias 201, Madrid.

NAZROO, J. (1995), "Uncovering gender differences in the use of marital violence: the effect of methodology", Sociology, n 29, pp. 475-499. Reimpreso en G. Allan (ed) (1999), The Sociology of the Family. A Reader, Blackwell, Oxford, pp. 129-148.

STETS, J.E. y M.A. STRAUS (1990), "The marriage license as hitting license: A comparison of assaults in clating, cohabiting and warried couples", en M.A. Straus y R.J. Gelles (eds.), Physical violence in American Families: Risk Factors and Adaptations to Violence in 8.145 Families, Nes Brunswick, NJ, Transaction.

STRAUS, M.A. (1999), "The controversy over domestic violence by women: A methodological, theoretical and sociology of science analysis", en X.B. Arriaga y S. Oskamp (eds), Violence in Intimate Relationships, Thousand Oaks, Sage.

VILLAVICENCIO, P. y J. SEBASTIÁN (1999), Violencia doméstica: su impacto en la salud fisica y mental de las mujeres, Madrid, Instituto de la Mujer. 